\title{
La tutoría en la UAA: lineamientos institucionales y expectativas de los estudiantes
}

Ana Cecilia Macías Esparza, Daniel Eudave Muñoz, María Guadalupe Muñoz Macías

\section{Introducción}

$\mathrm{L}$

as Instituciones de Educación Superior (IES) preocupadas por mejorar los procesos de formación profesional y atender de forma integral a sus estudiantes han diseñado distintas propuestas, una de ellas es el establecimiento de programas institucionales de tutoría que respondan a lo señalado por la Asociación Nacional de Universidades e Instituciones de Educación Superior (ANUIES) (1998) que recomienda la puesta en marcha de programas de tutoría a través de los cuales un profesor debidamente preparado apoye y aconseje a los alumnos a lo largo de su formación profesional.

En este contexto, la Universidad Autónoma de Aguascalientes (UAA) desde su Modelo Educativo Institucional (MEI) señala que la institución "concibe la educación como un proceso intencional y sistemático, cuya finalidad es la formación integral de la persona, esto es, el desarrollo pleno de todas sus facultades, posibilitando así la construcción de una sociedad más humana" (UAA, 2007: 4); las intenciones anteriores se concretizan en el Programa Institucional de Tutoría (PIT), cuya primera versión está fechada en 2003 y que fue revisado y actualizado en 2012, donde se establecen como funciones del tutor: favorecer el desarrollo personal y profesional y brindar apoyo académico; sin embargo, aun cuando existen documentos que norman e indican el tipo de acciones que se espera de los tutores, se sabe que la implementación de los programas de tutoría adquiere rasgos diversos en distintos contextos y programas, además de que se desconoce la percepción que los estudiantes tienen respecto a este apoyo institucional; en este sentido vale la pena rescatar lo que Sanz Oro (2005) indica al respecto: "hay cuestiones básicas que merecen nuestra atención: ¿Nuestras políticas y prácticas profesionales inciden positivamente en nuestros estudiantes?, ¿ven los estudiantes el ejercicio de la tutoría como algo normal y habitual y, por tanto, aumenta su uso y participación?, ¿qué y cómo debemos hacer para favorecer esta situación?" (p. 81). En este artículo se hace una reflexión sobre la propuesta institucional de tutoría y lo que los alumnos esperan de este apoyo.

\section{Acciones tutoriales: conceptualización, operación y expectativas}

Al hacer una revisión rápida de las definiciones que existen sobre las funciones del tutor encontramos una gran diversidad de concepciones, que van desde perfiles muy acotados centrados en el apoyo académico, hasta definiciones muy amplias que implican la atención de todas las esferas de desarrollo del individuo. Así, de acuerdo con Moreno (2011) el tutor debe ser el mediador entre el alumno y la comunidad universitaria, debe proporcionar atención personalizada, ofrecer soporte académico, psicológico, moral y emocional para ayudar al 
alumno a permanecer en la universidad y concluir sus estudios satisfactoriamente. Por su parte, Lobato, Del Castillo y Arbizu (2005) resaltan el papel del tutor como guía y seguimiento de los procesos de adquisición y maduración de los aprendizajes de cada estudiante. A su vez, Gallego (2006) señala las siguientes funciones: "Facilitar el desarrollo personal de los alumnos, supervisar los progresos de los estudiantes, hacer de puente entre los estudiantes y las autoridades académicas, presentarse como adulto responsable en el que los estudiantes pueden confiar e interceder delante de las autoridades académicas por los estudiantes" (p. 189).

Finalmente, Álvarez y González (2008) se dan a la tarea de recopilar las clasificaciones que, a lo largo de los años, han hecho varios autores como Boranat, Castaño y Ruiz, Del Rincón, Rodríguez Espinar, entre otros, encontrando claras coincidencias, pues si bien se utilizan diferentes denominaciones, todos los autores hacen mención de por lo menos tres tipos de tutoría: aquella relacionada con aspectos meramente académicos vinculados a cursos en particular; tutoría de carrera, en la que se atienden factores asociados con la inserción, permanencia y egreso de una institución de educación superior; y tutoría personal, que atiende aspectos y situaciones individuales que pueden ser de carácter psicológico, familiar, económico, sin dejar de reconocer la existencia de tutorías específicas para las actividades de práctica profesional o para el apoyo en trámites administrativos y burocráticos.

Considerando lo anterior, es claro que la tutoría es un concepto amplio que cubre distintos tipos de acciones y temáticas y que no hay definición conceptual suficientemente clara sobre el perfil y funciones del tutor, como señalan Giner y Puigardeu (2008) la construcción de un perfil profesional del tutor viene dada normalmente a través de la experiencia, lo que puede dificultar su capacidad como educador, y en este sentido es que se vuelve imprescindible una definición precisa en cada institución, pues de esta manera se puede orientar y facilitar el trabajo de todos los actores involucrados en la acción tutorial con la mejora consecuente en los resultados.

En nuestro país, la postura de la ANUIES es congruente con la tutoría de carrera (favorecer la permanencia y egreso exitoso de los estudiantes) sin dejar de lado la atención al ámbito personal.
En el caso de la UAA, el PIT 2012 define el siguiente objetivo de la acción tutorial: "apoyar y dar seguimiento a la trayectoria de los estudiantes de pregrado, a través de la atención académica y de los servicios educativos institucionales para incidir positivamente en los índices de rezago, reprobación, deserción y eficiencia terminal, elevando así la calidad de los procesos formativos a favor del desarrollo integral de los universitarios" (UAA, 2012: 16) y contempla dos vertientes: la incorporación a la vida universitaria y seguimiento al proceso de titulación.

Por tanto, en la UAA el énfasis está puesto en tutoría de carrera, además de que el PIT 2012 en sus lineamentos de operación delimita la acción tutorial al seguimiento de alumnos en situación de riesgo, señalando explícitamente que el tutor no debe ser mediador ante otros profesores y autoridades, no puede atender situaciones personales o psicológicas y que su rol no es el de informar sobre trámites administrativos, difiriendo así de las definiciones más amplias dadas por algunos autores como Moreno (2011) y Gallego (2006). Añadido a lo anterior, los resultados de algunos estudios realizados en la institución (Macías, 2005, Araiza, 2012) indican que, aun cuando hay diferencias en la implementación del PIT en los distintos Centros Académicos, las acciones realizadas por los tutores son generalmente informativas, más que formativas, la cuestión es si este tipo de acciones son las que requieren nuestros estudiantes.

Para conocer la postura de los alumnos al respecto, durante 2015-2016 se realizó un estudio en la UAA (Macías, Eudave, Carvajal, 2016) en el cual se indagaron las percepciones y expectativas de los estudiantes de ocho programas de licenciatura sobre las acciones tutoriales. En los resultados, los encuestados señalan que una acción realizada por sus tutores y que ha sido útil son las sesiones grupales para brindar información sobre créditos y requisitos de titulación, el campo laboral y actualizaciones profesionales, lo cual es congruente con lo encontrado en otros estudios sobre la función informativa de los tutores, pero que difiere de los lineamientos operativos del programa; además los estudiantes reconocen que el apoyo de los tutores ha sido útil para la atención de quejas y la resolución de problemas con profesores. Lo que llama particularmente la atención es que 
más de la mitad de los encuestados indica que les gustaría que se realizaran algunas acciones como parte de la tutoría, entre ellas: visitas a lugares de interés para la formación académica, canalización a áreas especializadas y estrategias personalizadas de acuerdo a necesidades individuales; así mismo, la mayoría de los alumnos que participaron en el estudio informan que les gustaría que en tutoría se abordaran temáticas vinculadas con estrategias de comprensión lectora, redacción de textos académicos, manejo de datos numéricos, técnicas de estudio, estrategias y dificultades de aprendizaje, organización del tiempo de estudio, horas de sueño, consumo de estimulantes, toma de decisiones sobre el estudio y la profesión, problemas psicológicos y económicos. A partir de estos datos podría decirse que los estudiantes demandan atención individual que va más allá de lo escolar, y si bien les interesan actividades y temas que puedan mejorar su desempeño académico existe también la necesidad de atender otro tipo de tópicos vinculados con el desarrollo personal y el establecimiento de hábitos de vida saludables.
Por otra parte, al ser cuestionados sobre el perfil que debieran tener los profesores en función de tutoría, los estudiantes señalan que deberían ser personas atentas, comprensivas, amables, que inspiren confianza y comprometidos con la actividad tutorial, con disponibilidad de tiempo y con constancia para llevar a cabo la acción tutorial, además de que les gustaría que realizaran actividades dinámicas, prácticas y recreativas, incorporando el uso de tecnologías y redes sociales y la atención individual.

Así pues, existen claras diferencias entre lo que señala el PIT como funciones y acciones que deben realizar los tutores y lo que los estudiantes informan como útil y deseable, ya que mientras en la institución se prioriza una atención remedial de tipo académica, de acuerdo con las respuestas, a los alumnos les gustaría más una atención preventiva, que atienda aspectos académicos, pero también personales, a cuyo cargo estén profesores que se muestran accesibles y que estén dispuestos a apoyarlos, acercándose más a la definición del tutor como adulto responsable que acompaña y apoya que dan Gallego (2006) y Moreno (2011).

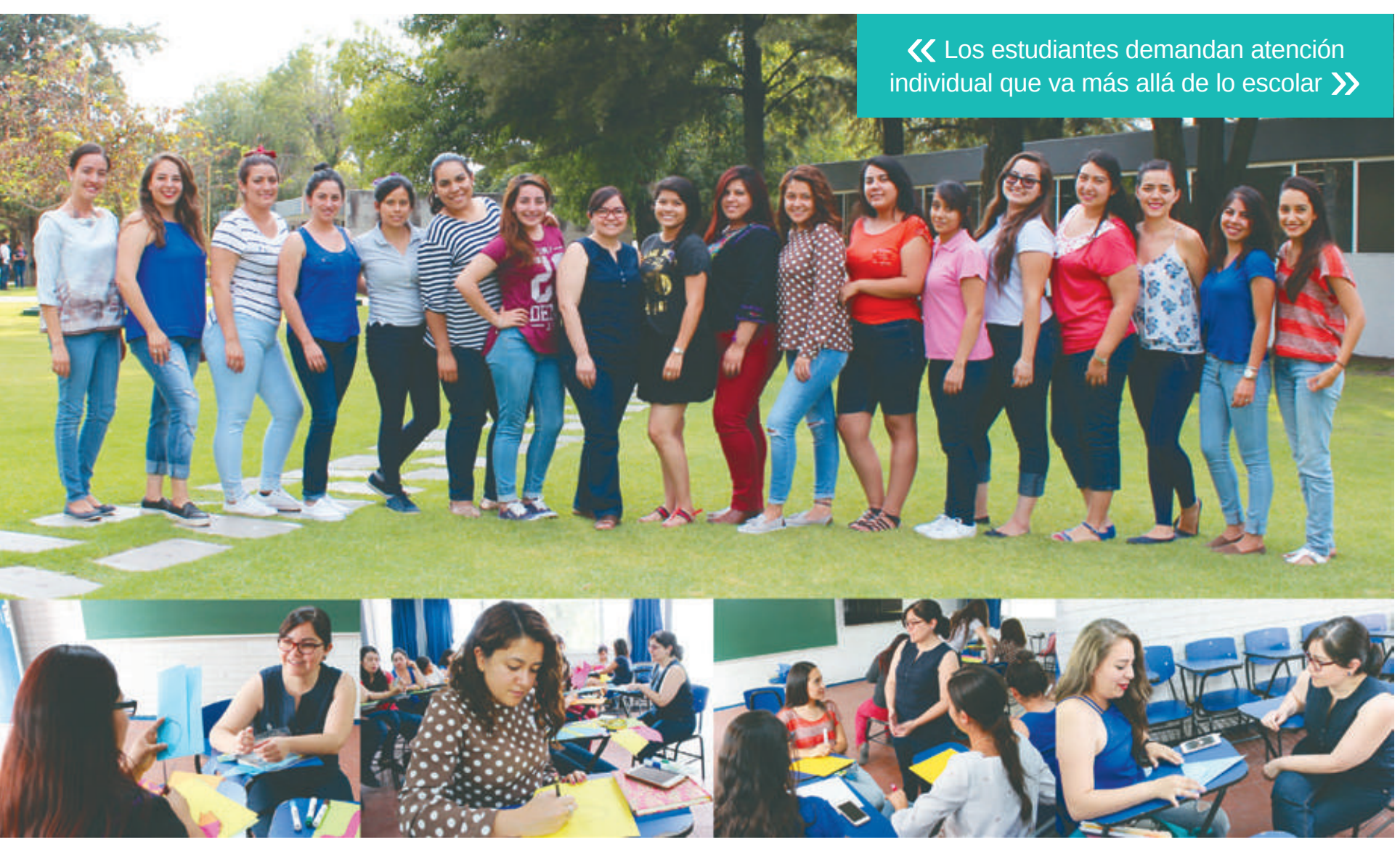




\section{Conclusión}

Los datos que se han presentado pueden servir para abrir la reflexión, en torno a lo que se requiere hacer en la institución para favorecer la formación integral de la que se habla en el MEI, es importante cuestionarse si es necesario un replanteamiento de la postura y enfoque del PIT, y considerar la factibilidad y posibles consecuencias del mismo.

En este sentido, este artículo más que dar respuestas abre nuevas interrogantes: ¿es factible cubrir las expectativas manifestadas por los estudiantes?, ¿qué condiciones institucionales se requieren?, ¿qué perfil y condiciones laborales debieran tener los profesores para brindar una tutoría de estas características?, con un cambio de esta naturaleza, ¿se lograrían mejores resultados?

Hay muchos aspectos por resolver en el campo de la tutoría, pero sin duda todos los involucrados en la implementación de este programa de apoyo tenemos un objetivo común: apoyar a los estudiantes para que logren ser mejores profesionistas y mejores personas.

\section{Fuentes de consulta}

Álvarez, P. y González M. (2008). Análisis y valoración conceptual sobre las modalidades de tutoría universitaria en el Espacio Europeo de Educación Superior. En Revista Interuniversitaria de Formación del Profesorado 22 (1), pp. 49-70.

ANUIES (1998). Programas institucionales de tutoría. Una propuesta de la ANUIES para su organización y funcionamiento en las Instituciones de Educación Superior. (2ª . Ed.). Disponible en http://www.anuies.mx/servicios/d_estratgicos/libros/lib42/000.htm

Araiza, M. (2012). Contribuciones del Programa Institucional de Tutoría al desarrollo académico de los estudiantes de pregrado en la Universidad Autónoma de Aguascalientes. Aguascalientes: UAA-Tesis de la Maestría en Investigación Educativa.

Gallego, S. (2006). La tutoría en la Educación Superior. En S. Gallego y J. Riart (Coord.), La tutoría y la orientación en el siglo XXI: Nuevas propuestas, pp. 185-196. Barcelona: Octaedro.

Giner, A. y Puigardeu, O. (2008). La tutoría y el tutor. Estrategias para su práctica. Barcelona: ICE-UB, Horsori Editorial.

Lobato, C., Del Castillo, L. y Arbizu F. (2005). Las representaciones de la tutoría universitaria en profesores y estudiantes: un estudio de caso. En International Journal of Psychology and Psychological Therapy 5 (2), pp. 148-168.

Macías, A., Eudave, D. y Carvajal, M. (2016). Tutoría y desarrollo de competencias académicas básicas. Percepciones y expectativas de estudiantes universitarios. Informe final del Proyecto de Investigación Educativa (PIE 15-1). Financiado por la Universidad Autónoma de Aguascalientes, Aguascalientes.

Macías, L. (2005). Una perspectiva de la tutoría en la Universidad Autónoma de Aguascalientes. Aguascalientes: UAA -Tesis de la Maestría en Investigación Educativa.

Moreno, T. (2011). El profesor-tutor universitario en el contexto de la sociedad del conocimiento. En Ducoing P. (Coord.); Tutoría y mediación I. México: UNAM- ISUE.

Sanz Oro, R. (2005). Integración del estudiante en el sistema universitario. La tutoría. Cuadernos de integración europea, 2 (1), pp. 69-95. UAA (2012). Marco Institucional de Formación Integral. Programa Institucional de Tutoría. Aguascalientes: UAA.

UAA (2007). Modelo Educativo Institucional. Correo Universitario, Sexta época (16). 15 de marzo de 2007. Aguascalientes: UAA. 